\title{
Helical Nanostructure of Achiral Silver p-Tolylacetylide Molecules
}

\author{
Ken Judai, ${ }^{1}$ Yoshikiyo Hatakeyama, ${ }^{1}$ and Junichi Nishijo ${ }^{2}$ \\ ${ }^{1}$ College of Humanities and Sciences, Nihon University, 3-25-40 Sakurajosui, Setagaya-ku, Tokyo 156-8550, Japan \\ ${ }^{2}$ School of Science and Engineering, Meisei University, 2-1-1 Hodokubo, Hino, Tokyo 191-8506, Japan \\ Correspondence should be addressed to Ken Judai; judai@chs.nihon-u.ac.jp
}

Received 8 August 2013; Accepted 4 September 2013

Academic Editor: Liqiang Jing

Copyright @ 2013 Ken Judai et al. This is an open access article distributed under the Creative Commons Attribution License, which permits unrestricted use, distribution, and reproduction in any medium, provided the original work is properly cited.

Silver $p$-tolylacetylide is an achiral molecule; however, its nanostructure has been found to consist of twisted nanoribbons. The twisted ribbon is a helicoid that combines translation and perpendicular rotation along the ribbon axis. A helix, a typical chiral structure, can be created by the aggregation of achiral molecules, and the recrystallization conditions control the twist of the nanoribbons. Therefore, the recrystallization controls the chirality.

\section{Introduction}

There are many known helical structures of various sizes. The winding staircase is a typical helical architectural feature on the macroscale. On the other hand, various important molecules in biology are microscopic helices, such as the DNA double helix and the alpha helix in proteins. Recent development in nanotechnology has produced helical species on the nanoscale. The aggregation of molecules can form nano-helical structures in supramolecular chemistry [1-5]. Helices coiled in the right-handed or left-handed direction can be formed, depending on the chirality of the constituent molecules. It is reasonable to assume that the chirality of a protein or structure comes from the chirality of the component amino acids (L-stereoisomers). Of course, a helix with the opposite direction of rotation will result from the use of amino acids of the opposite chirality (D-stereoisomers) [6] .

We have studied nanostructures of metal acetylide molecules $[7,8]$ and applied their nanostructures to gas sensors, catalysis, and so on [9-11]. In this study, we succeeded in producing a helical nanostructure from an achiral molecule of silver acetylide. The molecular structure of silver $p$-tolylacetylide is shown in Figure 1. The molecule contains no asymmetric carbon atoms and has mirror symmetry. Although silver $p$-tolylacetylide is an achiral molecule, the aggregated crystal is a twisted nanoribbon structure, a helicoid. The mechanism for aggregation from the achiral molecules to the chiral helical nanostructure has not been fully understood; however, primary results have been presented in order to reveal the creation of chirality with scanning electron microscopy (SEM) and powder X-ray diffraction measurements.

\section{Experiment}

Silver $p$-tolylacetylide was prepared by the reaction of silver(I) nitrate and 4-ethynyltoluene in the presence of triethylamine in an acetonitrile solvent. Since the crude product cannot be dissolved in most solvents, trimethylphosphine was added in a dichloromethane solution. The trimethylphosphine complex of silver $p$-tolylacetylene was then soluble in toluene or dichloromethane. Recrystallization was performed by 5-fold dilution of the saturated toluene solution by alcohol solvents. The phosphine was eliminated from silver $p$-tolylacetylide, and nanocrystals were obtained several days to one week later.

The precipitate of the recrystallization was analyzed by SEM (Keyence VE-9800) and powder X-ray diffraction measurements using $\mathrm{Cu}$ K-alpha emissions (Rigaku RINT2000). 


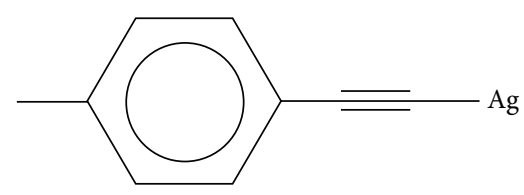

FIGURE 1: Molecular structure of silver $p$-tolylacetylide.

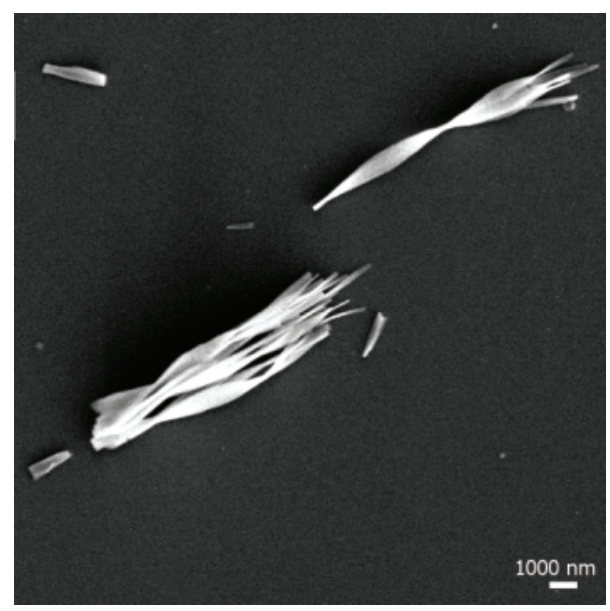

FIGURE 2: SEM image of recrystallized silver $p$-tolylacetylide in ethanol. The twisted nanoribbon structure is a helicoid.

\section{Results and Discussion}

Figures 2 and 3 show typical SEM images of recrystallized silver $p$-tolylacetylide in ethanol and 1-butanol, respectively. The polarity of the alcohol decreases gradually from methanol to ethanol, 1-propanol, and finally 1-butanol. The recrystallization rate also decreases from methanol to 1-propanol. The polarity of the alcohol and the recrystallization rate were roughly correlated to the shapes of the nanocrystals. Rapidly recrystallized solvents such as methanol and ethanol generated a twisted nanoribbon morphology, as shown in Figure 2. On the other hand, the nanocrystals in 1-propanol and 1-butanol contained few twisted nanoribbons, while the dominant morphology was a straight ribbon or wire nanostructure. Therefore, the nanomorphology of silver p-tolylacetylide can be controlled by changing the polarity of the solvent.

The twisted nanoribbon is a helicoid with a combination of translation and perpendicular rotation along the ribbon axis. The twisted ribbons are associated with a characteristic helix of coiled helical ribbons [12], and Ziserman et al. have reported the transition between the twisted ribbons and the coiled helical ribbons in amphipathic molecules [13]. As the helix is a typical chiral structure, a twisted ribbon can also be classified into left-handed or right-handed ribbon according to the direction of twist. Although the silver $p$-tolylacetylide nanostructure is a twisted ribbon that forms a chiral helix, surprisingly, the silver $p$-tolylacetylide molecule is an achiral molecule. The aggregation of achiral molecules forms a crystal of chiral helices. Since the component molecule is achiral, having mirror symmetry, the numbers of left-handed and right-handed twisted nanoribbons should be the same

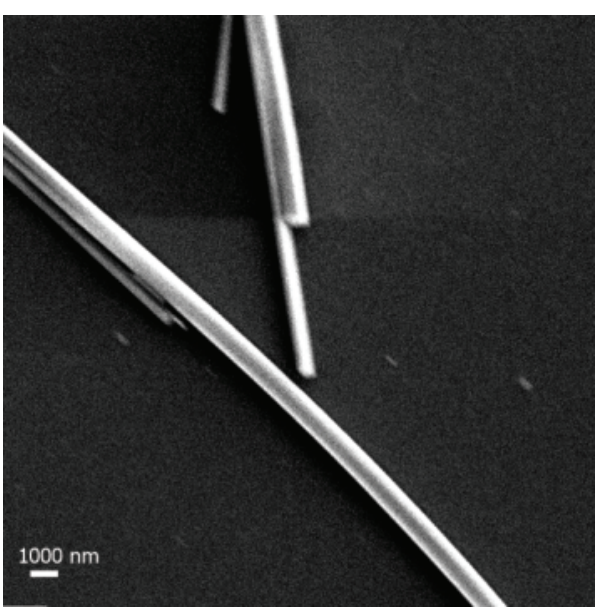

FIGURE 3: SEM image of recrystallized silver $p$-tolylacetylide in 1-butanol. The less polar solvent produced a non-helical straight nanoribbon structure.

(a racemic mixture). Actually, several tens of the crystals were analyzed by the SEM observations, and there were no difference between the numbers of left-handed and righthanded nanoribbons within statistical fluctuation. It should be noted that the emergence of chirality from the assembly of achiral molecules can be controlled by the recrystallization solvent. This nanosystem offers great advantages to the investigation of the origins of chirality, which could help answer one of the most important subjects in evolutionary biology, that is, homochirality.

In order to reveal the twisting mechanism, X-ray diffraction was performed for a powdered sample of silver p-tolylacetylide. Figure 4 illustrates the diffraction patterns of helical nanostructures from ethanol recrystallization and the non-helical structures from 1-butanol. There was no difference between the two patterns except for the peak widths. The peak positions and relative intensities were nearly identical to each other. The crystallinity generated in ethanol was less perfect, which caused slight peak broadening. The $\mathrm{X}$-ray diffraction observations indicate that the molecular packing matched up precisely between the helical and nonhelical crystals. Therefore, microscopic molecular orientation was not the driving force behind nanoribbon twisting.

At this stage, there is no direct evidence to prove the mechanism of nanoribbon twisting, but a small amount of crystal defects might be enough to twist a nanoribbon. The crystallinity in the helical sample was less perfect than that in the non-helical sample, according to the peak widths in the X-ray diffraction results. The SEM images of silver $p$-tolylacetylide supported this observation. The twisting crystals shown in Figure 2 were shorter than those shown in Figure 3. In other words, the non-helical samples not only had a straight structure, but also formed a much longer shape. In addition, twisting helices could be found with ribbon branches at several points. These SEM images revealed the less-perfect crystallinity of the twisting ribbon. The number of defects in the helical crystals should be higher than in the non-helical crystals, and these crystal defects 


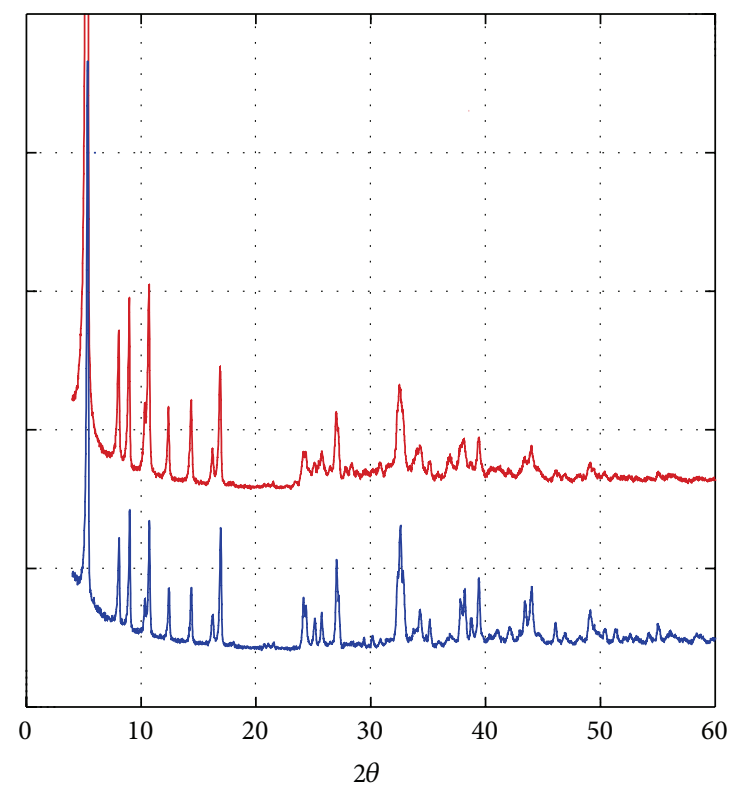

(a) $\mathrm{EtOH}$

(b) $\mathrm{BuOH}$

Figure 4: X-ray powder diffraction spectra of a helical sample recrystallized in ethanol (top) and a non-helical sample recrystallized in 1-butanol (bottom).

might twist the nanoribbon structures. Recently, Cui et al. have reported that acetylsalicylic acid crystals twisted in the presence of salicylic acid molecules as an impurity [14]. Silver $p$-tolylacetylide might twist in the same way.

In summary, we succeeded in controlling chirality by changing the recrystallization solvent. Even though silver p-tolylacetylide has mirror symmetry and is an achiral molecule, the aggregated nanocrystal takes, on a twisting nanoribbon morphology, a helicoidal structure. The chirality induced by the nano assembly can be controlled by changing the solvent for recrystallization. The crystal recrystallized in ethanol was a helical twisted nanoribbon, while that recrystallized in 1-butanol resulted in a non-helical straight ribbon structure. The chirality as the helix can emerge from achiral molecules, and then this system may be suitable for investigating the origins of homochirality in biology.

\section{Conflict of Interests}

The authors declare that there is no conflict of interests.

\section{Acknowledgment}

This work was supported in part by the Nanotechnology Excellence, Nihon University “N.” Research Project.

\section{References}

[1] Y. Shinozaki, G. Richards, K. Ogawa et al., "Double helices of a pyridine-appended zinc chlorophyll derivative," Journal of the American Chemical Society, vol. 135, pp. 5262-5265, 2013.
[2] S. Yagai, M. Yamauchi, A. Kobayashi et al., "Control over Hierarchy levels in the self-assembly of stackable nanotoroids," Journal of the American Chemical Society, vol. 134, pp. 1820518208, 2012.

[3] L. Ziserman, A. Mor, D. Harries, and D. Danino, "Curvature instability in a chiral amphiphile self-assembly," Physical Review Letters, vol. 106, no. 23, Article ID 238105, 4 pages, 2011.

[4] S. Matsushita, M. Kyotani, and K. Akagi, "Hierarchically controlled helical graphite films prepared from iodine-doped helical polyacetylene films using morphology-retaining carbonization," Journal of the American Chemical Society, vol. 133, no. 44, pp. 17977-17992, 2011.

[5] M. Goh, M. Kyotani, and K. Akagi, "Highly twisted helical polyacetylene with morphology free from the bundle of fibrils synthesized in chiral nematic liquid crystal reaction field," Journal of the American Chemical Society, vol. 129, no. 27, pp. 8519-8527, 2007.

[6] M. A. Shmilovici, K. Mandal, Z. P. Gates, N. B. Philips, M. A. Weiss, and S. B. H. Kent, "Fully convergent chemical synthesis of ester insulin: determination of the high resolution X-ray structure by racemic protein," Journal of the American Chemical Society, vol. 135, pp. 3173-3185, 2013.

[7] K. Judai, J. Nishijo, and N. Nishi, "Self-assembly of copper acetylide molecules into extremely thin nanowires and nanocables," Advanced Materials, vol. 18, no. 21, pp. 2842-2846, 2006.

[8] N. Nishi, J. Nishijo, O. Oishi, and K. Judai, "Facile and massproducible fabrication of one-dimensional Ag nanoparticle arrays," Chemistry of Materials, vol. 19, no. 19, pp. 4627-4629, 2007.

[9] K. Judai, S. Numao, A. Furuya, J. Nishijo, and N. Nishi, "Increased electric conductance through physisorbed oxygen on copper nanocables sheathed in carbon," Journal of the American Chemical Society, vol. 130, no. 4, pp. 1142-1143, 2008.

[10] S. Numao, K. Judai, J. Nishijo, K. Mizuuchi, and N. Nishi, "Synthesis and characterization of mesoporous carbon nanodendrites with graphitic ultra-thin walls and their application to supercapacitor electrodes," Carbon, vol. 47, no. 1, pp. 306-312, 2009.

[11] K. Judai, S. Numao, J. Nishijo, and N. Nishi, "In situ preparation and catalytic activation of copper nanoparticles from acetylide molecules," Journal of Molecular Catalysis A, vol. 347, no. 1-2, pp. 28-33, 2011.

[12] S. Armon, E. Efrati, R. Kupferman, and E. Sharon, "Geometry and mechanics in the opening of chiral seed pods," Science, vol. 333, no. 6050, pp. 1726-1729, 2011.

[13] L. Ziserman, H.-Y. Lee, S. R. Raghavan, A. Mor, and D. Danino, "Unraveling the mechanism of nanotube formation by chiral self-assembly of amphiphiles," Journal of the American Chemical Society, vol. 133, no. 8, pp. 2511-2517, 2011.

[14] X. Cui, A. L. Rohl, A. Shtukenberg, and B. Kahr, "Twisted aspirin crystals," Journal of the American Chemical Society, vol. 135, pp. 3395-3398, 2013. 

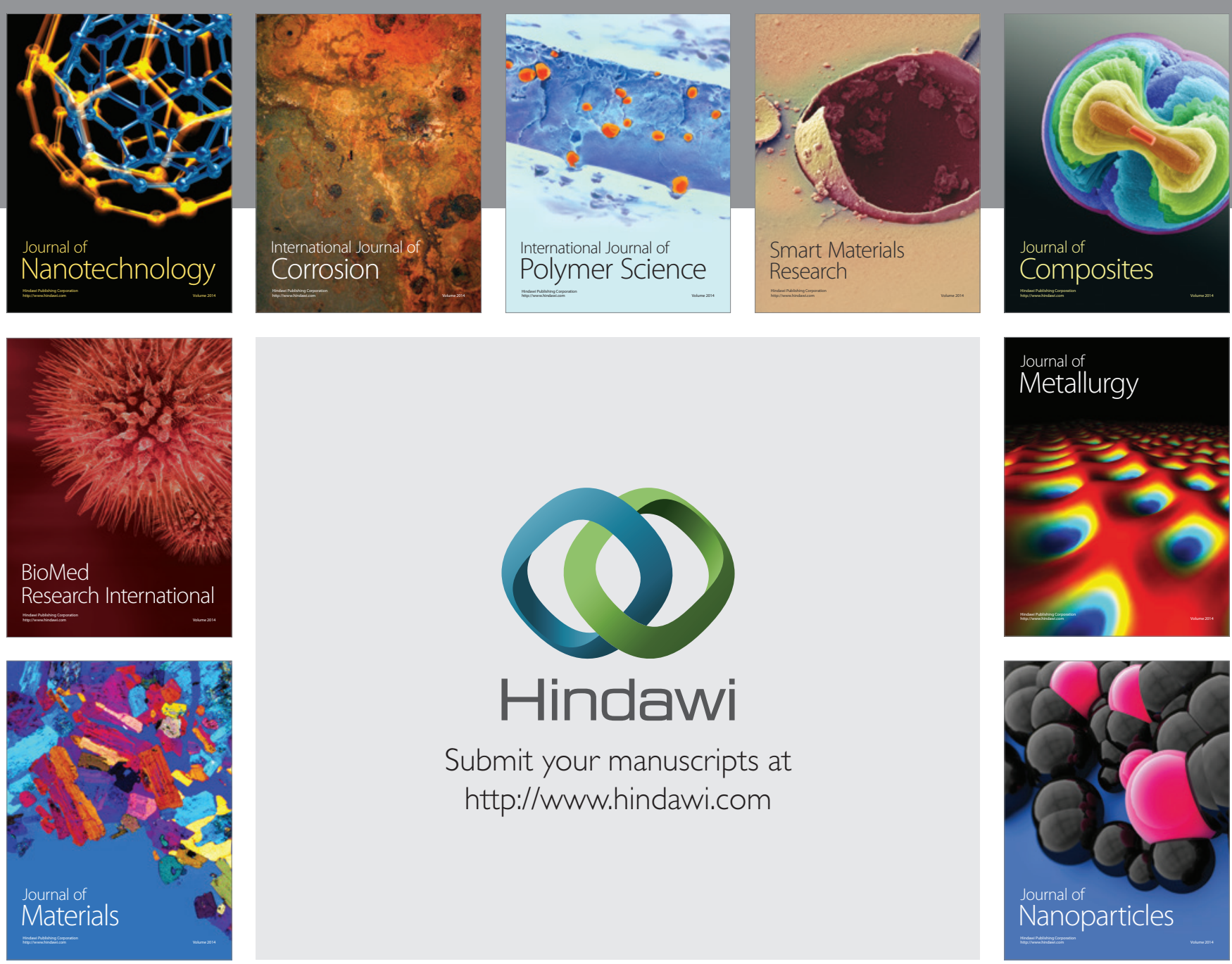

Submit your manuscripts at http://www.hindawi.com
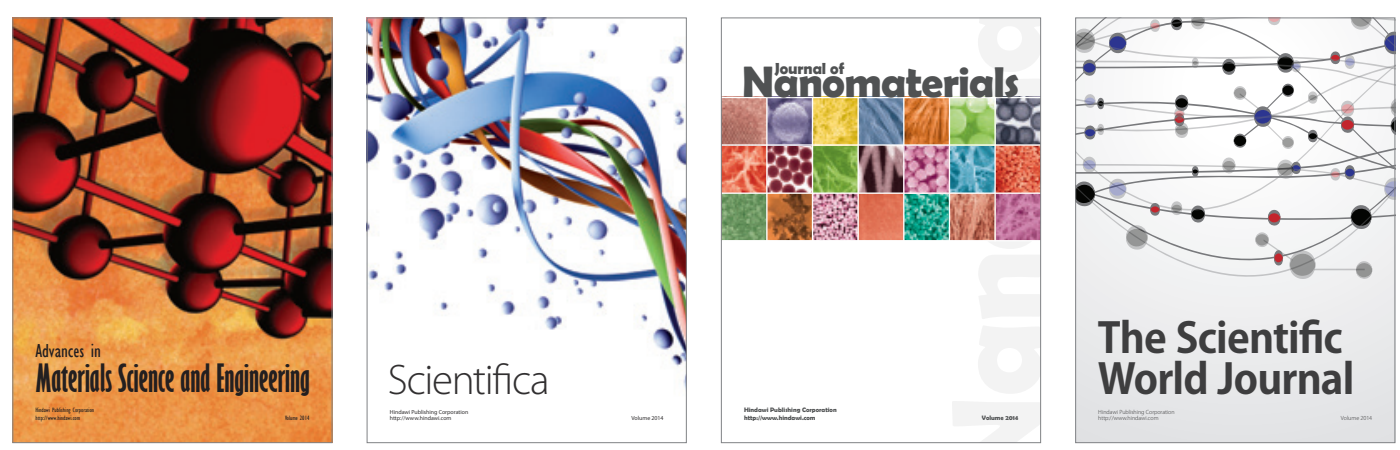

\section{The Scientific World Journal}
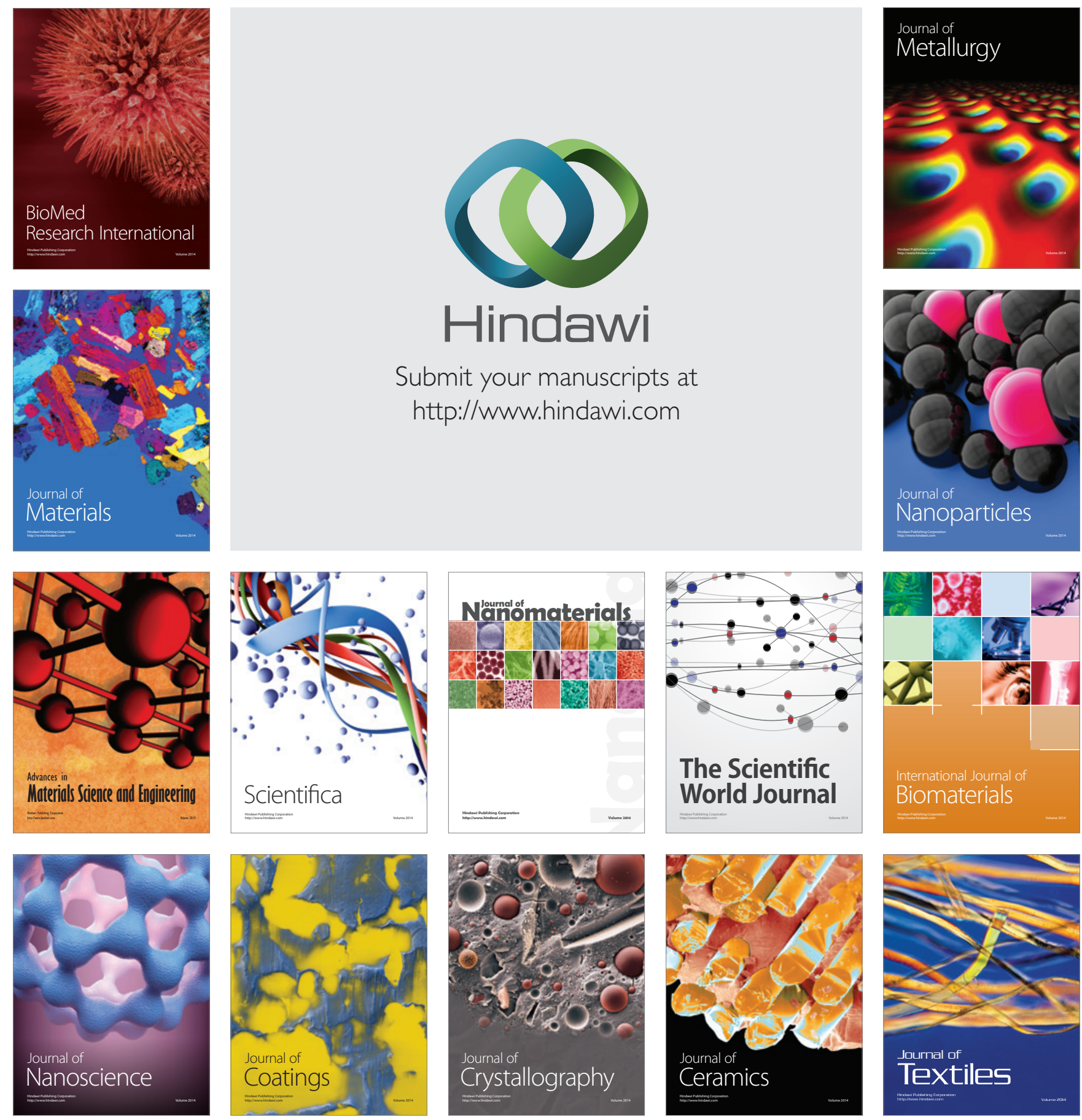